\title{
Integrated Modeling of the TESLA X-ray FEL
}

\author{
T. Limberg and Ph. Piot *, DESY, Hamburg, Germany
}

\section{Abstract}

The TESLA linear collider incorporates an X-ray SASE FEL which demands challenging electron beam parameters (typically transverse emittance of $1.6 \mathrm{~mm}$ - $\mathrm{mrd}$ and peak current of $5 \mathrm{kA}$ ). For a realistic electron beam distribution at the entrance of the undulator, tracking has to be done from the cathode through the whole accelerator. Non-Gaussian beam profiles have to be taken into account as well as nonlinear effects such as space-charge, coherent synchrotron radiation field and wake fields. We have done this with several codes: Astra, for the low energy part $(<100 \mathrm{MeV}), \mathrm{TraFiC}^{4}$ for the bunch compressor chicanes where CSR influences the particle trajectories and the code Elegant for the $6 \mathrm{D}$ tracking with wake fields in the linacs and transport lines. The so-generated electron phase space density at the undulator entrance can then passed to SASE FEL simulation codes. Results of this integrated modeling is discussed.

\section{INTRODUCTION}

The TESLA linear collider [1] is foreseen to integrate an X-ray FEL user facility producing photons with wavelengths ranging from 1 to 25 Angstroms. The required electron beam parameters at the entrance of the undulators are: for a beam energy range between 13 and $50 \mathrm{GeV}$, a peak current of $5 \mathrm{kA}$, and a transverse emittance less than $1.6 \mathrm{~mm}$-mrd must be provided. The transverse emittance requirement is specified for a longitudinal bunch slice which length is comparable to the cooperation length; in the present case less than $1 \mu \mathrm{m}$. The FEL

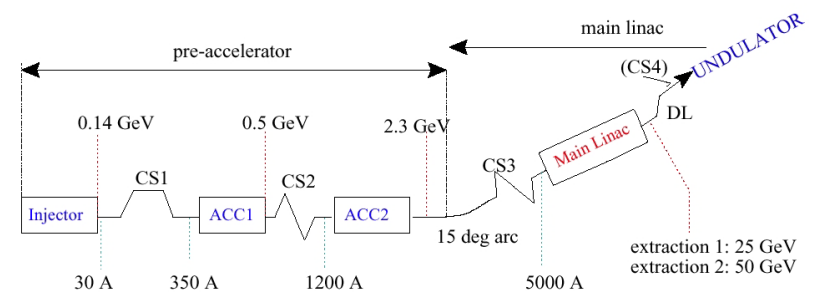

Figure 1: Schematic rendition of the TESLA X-ray FEL driver-accelerator.

driver-accelerator (see Fig. 1) has its own pre-accelerator, necessary to produce the required high brightness beam: it incorporates a photo-injector [2] followed by two compressors (CS1, CS2) inter-spaced by accelerating sections (ACC1, ACC2). At $\sim 2.3 \mathrm{GeV}$, the beam is injected into

\footnotetext{
*PIOT@MAIL.DESY.DE
}

the TESLA main linac after traveling through a $15^{\circ} \operatorname{arc}^{1}$ and third compressor (CS3). The momentum compaction of the $15^{\circ}$ arc is $R_{56}=+8 \times 10^{-4}$; under the nominal time-energy correlation of the incoming beam, this arc will slightly decompress the bunch $\left(\Delta \sigma_{z} \simeq 10 \mu \mathrm{m}\right)$. The settings of the linac and bunch compressors are gathered in Table 1. The electron beam can be extracted from the

\begin{tabular}{lccc}
\hline \hline Linacs: & & & \\
parameters & ACC1 & ACC2 & Main Linac \\
\hline Grad $(\mathrm{Mv} / \mathrm{m})$ & 25 & 25 & 18 \\
RF phase $\left(^{\circ}\right)$ & -40.0 & -7.5 & 0.0 \\
phase adv./cell $\left(^{\circ}\right)$ & - & 90 & 90 \\
input $E(\mathrm{GeV})$ & 0.14 & 0.44 & 2.22 \\
final $E(\mathrm{GeV})$ & 0.44 & 2.22 & 24.6 \\
input $\delta p / p(\%)$ & 1.60 & 1.01 & 0.18 \\
final $\delta p / p(\%)$ & 1.01 & 0.18 & 0.04 \\
$\sigma_{z}(\mu \mathrm{m})$ & 300 & 90 & 40 \\
\hline Compressors: & & & \\
parameters & $\mathrm{CS} 1$ & $\mathrm{CS} 2$ & $\mathrm{CS} 3$ \\
\hline type & standard & $\mathrm{S}-\mathrm{chic}$ & $\mathrm{S}-\mathrm{chic}$ \\
Angles $\left({ }^{\circ}\right)$ & 10 & $2.4 \mid 4.8$ & $3 \mid 6$ \\
$R_{56}(\mathrm{~mm})$ & -92.4 & -22.9 & -35.9 \\
$T_{556}(\mathrm{~mm})$ & 145.7 & 34.5 & 53.9 \\
$E(\mathrm{GeV})$ & 0.14 & 0.44 & 2.22 \\
$\delta p / p(\%)$ & 1.60 & 1.01 & 0.18 \\
input $\sigma_{z}(\mu \mathrm{m})$ & 1800 & 300 & 90 \\
final $\sigma_{z}(\mu \mathrm{m})$ & 300 & 90 & 40 \\
final $\hat{I}(\mathrm{~A})$ & 350 & 1200 & 5800 \\
\hline \hline
\end{tabular}

Table 1: Linac and bunch compressor parameters. A detailed description of the bunch compressors can be found in Ref [10] (for the "standard"-type) and Ref [11] (for the "S-chic"-type).

main linac at two extraction points $(\mathrm{E}=13 \div 27 \mathrm{GeV}$ and $\mathrm{E}=20 \div 50 \mathrm{GeV}$ ) and transported to the undulator sections via a transfer line ( $\sim 12 \mathrm{~km}$ long). The extraction is done with pulsed bending magnets, arranged in a "dog-leg" (DL) configuration of insignificant momentum compaction. Optionally the beam can be further compressed by a F0D0-type compressor (CS4) making use of the time-energy correlation induced by geometric wakes [3] of the accelerating structures.

The principal effects that degrade the beam in the TESLA X-FEL accelerator are: single bunch self interac-

\footnotetext{
${ }^{1}$ This arc is required to accommodate a special geometry of the layout that supports the "TERA" option where bunches from TESLA could collide with HERA protons
} 
tion via (1) coherent synchrotron radiation (CSR) fields in bunch compressors section, (2) geometric wake fields in the TESLA cavities, and (3) resistive wake fields in transfer lines and other straight sections. CSR effects are calculated in a self-consistent manner using the program $\mathrm{TraFiC}^{4}$ [5], while the short range wakes are treated via a "kick approximation", using the code Elegant [6]. In the present study, we only include the longitudinal monopole mode of the wakes using the Green functions provided in Ref. [7] and Ref. [8], respectively for the geometric and resistive wakes.

\section{SIMULATION TECHNIQUE}

The low energy $(E<0.14 \mathrm{GeV})$ beamline has been optimized with the multi-particle code Astra [4, 2], which is based on a space charge mesh-type algorithm. The transport through the straight sections is modeled with the single particle code Elegant [6], a six-dimensional code that includes an approximative treatment of the wake fields. Tracking through the magnetic bunch compressors and other dispersive sections is performed with the multiparticle code TraFiC ${ }^{4}$. Phase space distributions, $\Phi(\mathbf{x} ; z)$, can be passed between Astra and Elegant without any manipulation. However, this is not the case for the code $\mathrm{TraFiC}^{4}$, where the use of thousands of field generating particles would be prohibitive with respect to CPU-time.

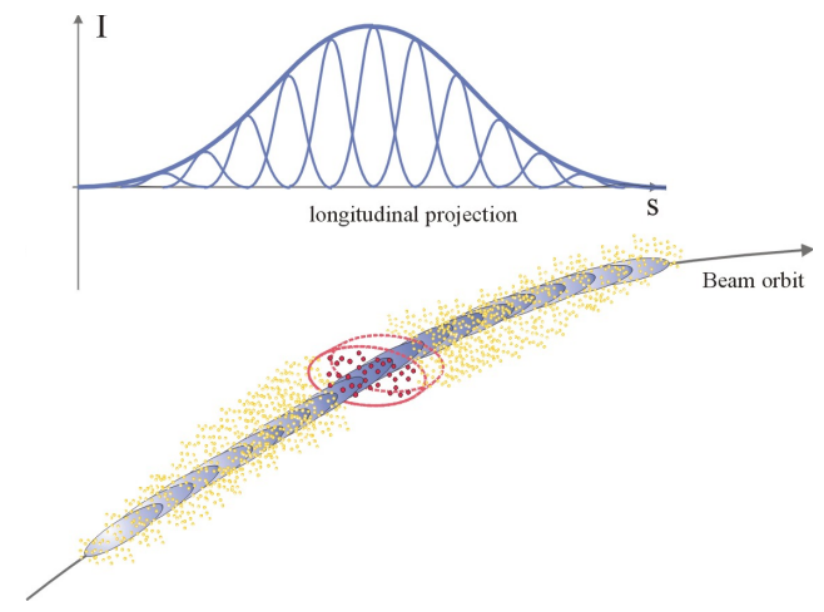

Figure 2: The different particles ensembles in $\mathrm{TraFiC}^{4}$. The full ellipses represent the sub-bunches of $\Phi_{\text {gen }}$ ensemble. The dots represent the witness ensembles $\Phi_{\text {opt }}$ (at the bunch center - outlined here with the red ellipses) and $\Phi_{\text {sampling }}$ (surrounding the whole bunch).

$\mathrm{TraFiC}^{4}$ allows the input of various ensembles (see Fig. 2): (1) a generating ensemble, $\Phi_{\text {gen }}$, (2) an optic ensemble,$\Phi_{\text {opt }}$, and (3) a sampling ensemble, $\Phi_{\text {sampling }}$. While $\Phi_{g e n}$ is used to compute the electro-magnetic field, $\Phi_{\text {opt }}$ is generally used to study the influence of the field on a center slice (a 6D-Gaussian density distribution internally generated populates this ensemble), and $\Phi_{\text {sampling }}$ allows to study the impact of the field on "real", user-specified, incoming phase space. For copy $\Phi_{s g n}$, of the generating ensemble $\Phi_{g e n}$, the orbit changes due to the CSR field are applied and correlated emittance growth is evaluated. In the self-consistent mode, fields of the "gen" and "sgn" ensembles act onto each other. created to reflect the effect of the self-interaction on the generating bunch itself. The CPU time is $\propto N_{\text {gen }} \times\left(N_{\text {gen }}+N_{\text {opt }}+N_{\text {sampling }}\right)$ where $N$ is the number of sub-bunches (or macroparticles) in each ensemble.

A "condensed" copy of the incoming phase space is used as the $\Phi_{g e n}$ ensemble; it typically contains $\sim 300-500$ macroparticles. The whole incoming phase space, that can consists of thousands of macroparticles, is condensed to 300-500 macroparticles to set up the $\Phi_{\text {gen }}$ ensemble. The condensed phase space is generated as follows (for further study see Ref. [12]):

$$
\begin{gathered}
\Phi_{g e n}(\mathbf{x} ; z)=\sum_{i=1}^{N_{g e n}} q_{i} \times \frac{e^{-\left(s-s_{i}\right)^{2} /\left(2 \sigma_{z}^{2}\right)}}{\sqrt{2 \pi} \sigma_{z}} \delta\left(\delta-\delta_{i}\right) \times \\
\frac{e^{-\left[\left(x-x_{i}\right)^{2}+\left(y-y_{i}\right)^{2}\right] /\left(2 \sigma_{r}^{2}\right)}}{\sqrt{2 \pi} \sigma_{r}} \times \delta\left(x^{\prime}-x_{i}^{\prime}\right) \delta\left(y^{\prime}-y_{i}^{\prime}\right),
\end{gathered}
$$

where $\delta(\ldots)$ stands for the Dirac-function. The charge $q_{i}$ and first order moments are obtained by binning the incoming phase space distribution. The sub-bunches are 3D Gaussian charge distribution with fixed length, $\sigma_{z}$, and radius, $\sigma_{r}$, which are user-specified parameters. However, we smooth offsets and charge variation with polynominal fits to avoid numerical noise.

A few meters downstream of the last dipole, to account for co-propagating transient CSR fields, the $\Phi_{\text {sampling }}$ ensemble is then passed, without any operation, to Elegant or Astra (depending on the beam physics dominating the downstream beamline).

\section{START-TO-END SIMULATION OF THE X-RAY FEL}

In this section we present an example of beam dynamics simulation for the TESLA X-FEL accelerator; the beam is assumed to be extracted from the main linac at the first extraction point at an energy of $E \simeq 24.6 \mathrm{GeV}$. The injector simulation and optimization is discussed elsewhere [2] and the phase space density obtained with Astra at the injector front end is our starting point, i.e. $2 \mathrm{~m}$ upstream of CS1. The critical point for avoiding un-tolerable emittance growth is to properly match the beam into the bunch compressors [9]. The typical $\beta$-functions along the whole accelerator (excluding the photo-injector) are plotted in Fig. 3. The evolution of the longitudinal phase space and charge density profile is presented after each compression stage in Fig. 4. This figure demonstrates the highly nonGaussian character of the distribution that arises from the input distribution but also via CSR and wake fields along the beamline. For a $1 \mathrm{nC}$ Gaussian charge density, a peak current of $5 \mathrm{kA}$ would correspond to $\sigma_{z}=25 \mu \mathrm{m}$, while in the present case, for the same peak current achieved, we 


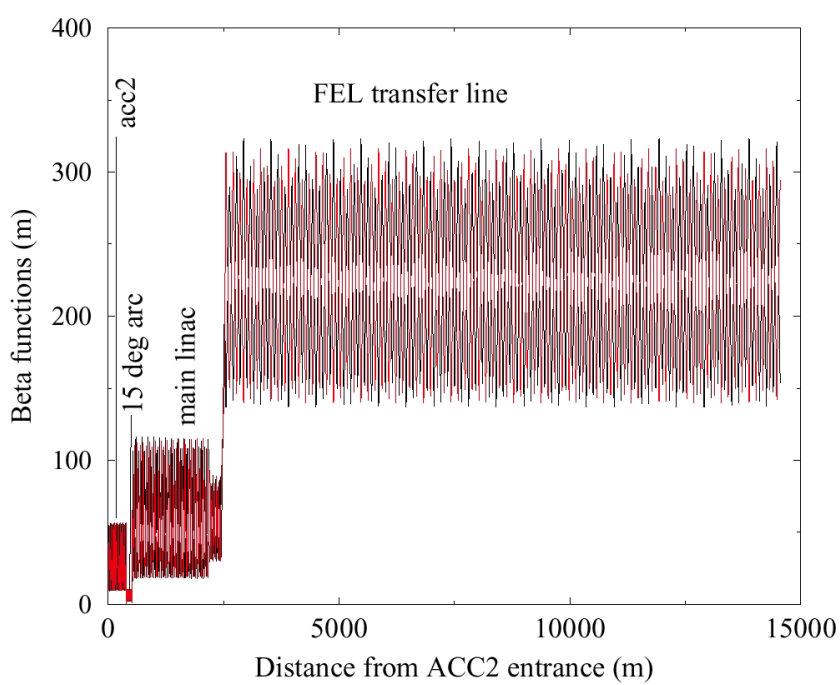

Figure 3: Lattice along the whole linac, starting from ACC 2 entrance up to end of the transfer line.

have an rms length of $40 \mu \mathrm{m}$. Note that, without using a $3^{\text {rd }}$ harmonic rf-section [2] in the injector, the longitudinal profiles would be even more distorted.

Fig. 5 shows peak current and transverse emittance (within a correlation length) along the bunch. Regions where the peak current is above $\sim 3 \mathrm{kA}$ and the transverse emittance below $\sim 1.6 \mathrm{~mm}$-mrd will reach saturation in the $\mathrm{X}$-FEL undulator. We estimate, for this set up, that about $40 \%$ of the beam intensity contributes fully to the lasing process.

\section{SUMMARY}

Using the three codes, Astra, Elegant, and TraFiC ${ }^{4}$, we have performed a thorough integrated modeling of the TESLA X-ray FEL, from the photocathode surface up to the undulator entrance. Our present efforts are (1) to automate the procedure using a high level interfacing between the codes and (2) to increase the simulation speed. This latter point is about to be solved with the availability of MPP versions of $\mathrm{TraFiC}^{4}$ and Astra along with an efficient passing of phase space density to $\mathrm{TraFiC}^{4}$ code [12].

\section{REFERENCES}

[1] TESLA TDR, rep. TESLA-01-23, DESY-HH (2001)

[2] Flöttmann K., et al. “The TESLA X-FEL injector”, PAC2001

[3] Brinkmann R., et al., proc. of PAC'97, pp. 1572-1574 (1997)

[4] Flöttmann K., AstraUser Manual http://www.desy.de/ mpyflo/Astra_dokumentation/

[5] Dohlus M., et al., NIM A 445, pp. 338-342 (2000)

[6] Borland M., report LS-287, APS/ANL (2000)

[7] Novokhatski A., et al., report TESLA-99-16 (1999)

[8] Bane K.L.F, et al., report SLAC-PUB-95-7074 (1995)
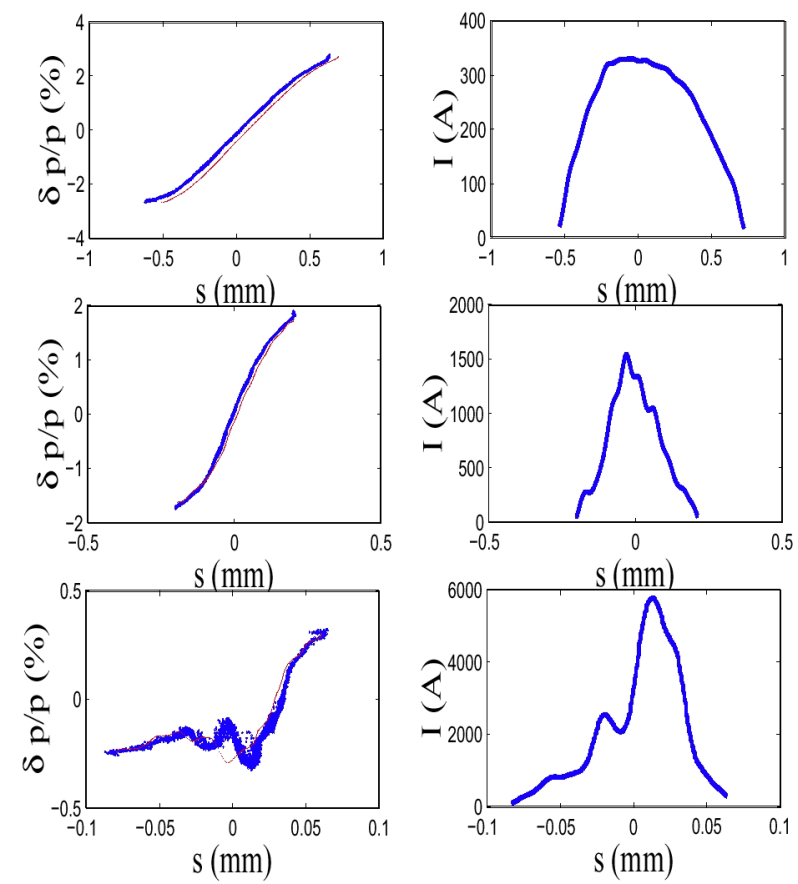

Figure 4: Longitudinal phase space $(s, \delta p / p)$ and charge density $\hat{I}(s)$ downstream of CS1, CS2, and CS3 (from top to bottom row).

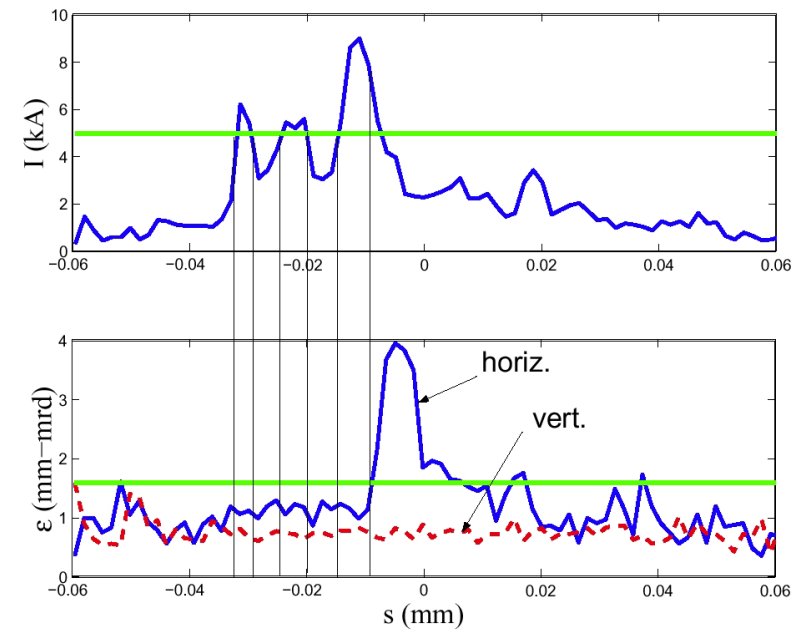

Figure 5: Peak current (top) and transverse emittances (bottom) within longitudinal slice of $1.5 \mu \mathrm{m}$ width along the bunch. The horizontal line indicates the minimum required peak current and maximum tolerated emittances. The vertical lines delimits area of lasing with $\varepsilon \leq 1.6 \mathrm{~mm}$ mrd and $I>5 \mathrm{kA}$.

[9] Limberg T. et al., "Optics optimization for multistage compression" to be published as a TESLA-FEL report (2001)

[10] Limberg T. et al., proc. of FEL2000 in press

[11] Loulergue A. et al., proc. of EPAC2000, pp. $754-756$ (2000)

[12] Dohlus M., et al.,"Beam dynamics simulations in magnetic bunch compressors using realistic particle distributions", submitted to FEL2001 